\title{
The Korteweg-de Vries equation and water waves. Part 2. Comparison with experiments
}

\author{
By JOSEPH L. HAMMACK \\ W. M. Keck Laboratory of Hydraulics and Water Resources, \\ California Institute of Technology, Pasadena \\ AND HARVEY SEGUR \\ Department of Mathematies, Clarkson College of Technology, \\ Potsdam, New York
}

(Received 10 September 1973)

The Korteweg-de Vries ( $\mathrm{KdV}$ ) equation is tested experimentally as a model for moderate amplitude waves propagating in one direction in relatively shallow water of uniform depth. For a wide range of initial data, comparisons are made between the asymptotic wave forms observed and those predicted by the theory in terms of the number of solitons that evolve, the amplitude of the leading soliton, the asymptotic shape of the wave and other qualitative features. The $\mathrm{KdV}$ equation is found to predict accurately the number of evolving solitons and their shapes for initial data whose asymptotic characteristics develop in the test section of the wave tank. The accuracy of the leading-soliton amplitudes computed by the $\mathrm{KdV}$ equation could not be conclusively tested owing to the viscous decay of the measured wave amplitudes; however, a procedure is presented for estimating the decay in amplitude of the leading wave. Computations suggest that the $K d V$ equation predicts the amplitude of the leading soliton to within the expected error due to viscosity $(12 \%)$ when the non-decayed amplitudes are less than about a quarter of the water depth. Indeed, agreement to within about $20 \%$ is observed over the entire range of experiments examined, including those with initial data for which the non-decayed amplitudes of the leading soliton exceed half the fluid depth.

\section{Introduction}

The equation

$$
f_{\tau}+6 f f_{r}+f_{r r r}=0
$$

was derived by Korteweg \& de Vries (1895) to describe the propagation in one direction of long water waves of moderate amplitude in shallow water. A method of solving (1) as an initial-value problem was discovered by Gardner et al. (1967) and requires that the initial data satisfy the following conditions:

and

$$
\int_{-\infty}^{\infty} \sum_{n=0}^{3}\left[\frac{\partial^{n} f(r ; 0)}{\partial r^{n}}\right]^{2} d r<\infty
$$


The outstanding feature of this model is that any initial data satisfying (2) and (3) finally evolve into a finite set of stable positive waves, called solitons (Zabusky \& Kruskal 1965), which are followed by a dispersive train of oscillatory waves (tail). The parameters required to describe completely this asymptotic solution can be obtained directly from the initial data; hence, a step-by-step integration of the differential equation can be avoided. Methods of determining this asymptotic solution were discussed in part 1 of this study (Segur 1973).

The purpose of part 2 is to examine the validity of (1) as a model equation for water waves by comparing the observed evolution of waves in a long tank of uniform depth with the asymptotic behaviour predicted by (1). Comparisons between experiment and theory are made over a wide range of initial data; features of the asymptotic wave behaviour such as the shape of the final wave form, the number of solitons that evolve and the amplitude of the leading soliton are examined quantitatively. Qualitative comparisons are also made for certain general features of the asymptotic solution. The method of wave generation in the laboratory is similar to the generation of tsunamis in the ocean by the vertical deformation of the sea bed; hence, the results of this study may be particularly relevant to the engineering problem of tsunami propagation.

Comparisons of the Korteweg-de Vries equation with experiments have also been made by Zabusky \& Galvin (1971) for shallow-water waves and by Hershkowitz, Romesser \& Montgomery (1972) for ion acoustic waves in a plasma. The effect of each of these studies was to establish the existence of solitons as an essential ingredient in the description of long waves of moderate amplitude propagating in their respective media. The present study seeks to test the ability of the KdV model to predict quantitatively the asymptotic form of the wave that evolves from arbitrary initial conditions.

\section{Review of the theory}

Consider an inviscid fluid of constant density which rests on a horizontal and impermeable bed of infinite lateral extent as shown in figure 1 . The fluid is subject to a vertical gravitational force $g$ and possesses a free surface along which the pressure is constant and surface-energy effects are negligible. Required are the two-dimensional and irrotational motions which are bounded everywhere and evolve from a given initial distribution of velocities and free-surface deformation, i.e., the classical water-wave problem. To derive the Korteweg-de Vries equation from the governing equations of this problem the following assumptions are necessary: (i) long waves for which the square of the ratio of the water depth $h$ to a characteristic horizontal length scale $l$ is small, i.e., $(h / l)^{2} \ll 1$, (ii) small amplitude waves for which the ratio of a typical wave amplitude $\left|\eta_{0}\right|$ to the water depth $h$ is small, i.e. $\left|\eta_{0}\right| / h \ll 1$, and (iii) that these two parameters are approximately equal, i.e., $\epsilon \equiv\left|\eta_{0}\right| / h=O\left[(h / l)^{2}\right] \ll 1$. These assumptions have been used by many authors (see, for example, Korteweg \& de Vries 1895; Benney \& Luke 1964) to derive (1). At the lowest order in $\epsilon$ the governing equations yield the linearized theory for long waves:

$$
\eta_{t}+q \eta_{x}+h u_{x}=0, \quad u_{t}+q u_{x}+g \eta_{x}=0, \quad v=-(y+h) u_{x}
$$




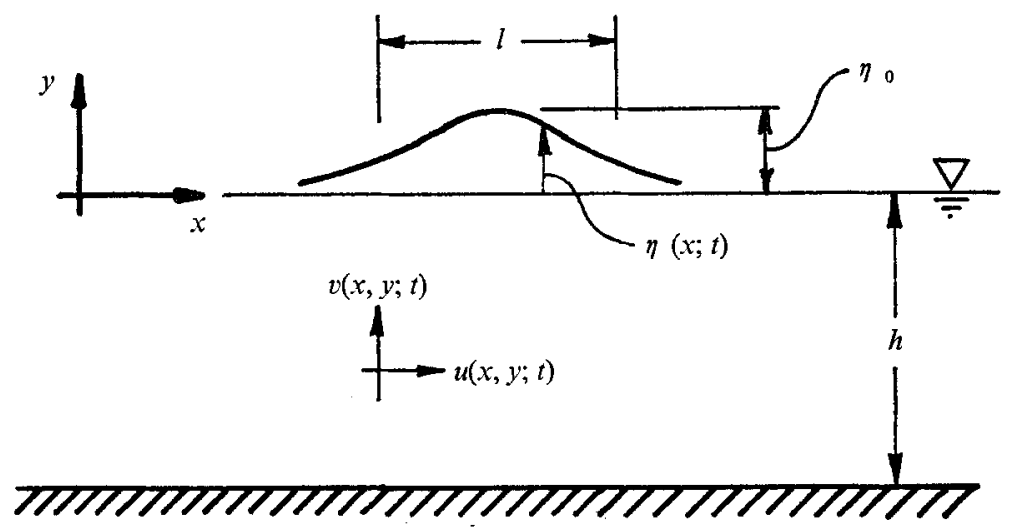

Figure 1. Definition sketch of co-ordinate system.

where $q$ is the (constant) translational velocity of the observer. For the present development the translational velocity may be taken as zero; the well-known solution of (4) then becomes

$$
\left.\begin{array}{l}
\eta(x ; t)=F_{1}\left(x-(g h)^{\frac{1}{2}} t\right)+F_{2}\left(x+(g h)^{\frac{1}{2}} t\right), \\
u(x ; t)=(g / h)^{\frac{1}{2}}\left[F_{1}\left(x-(g h)^{\frac{1}{2}} t\right)-F_{2}\left(x+(g h)^{\frac{1}{2}} t\right)\right] .
\end{array}\right\}
$$

At this order an arbitrary initial disturbance decomposes into two sets of waves travelling in opposite directions with a speed $(g h)^{\frac{1}{2}}$; these waves propagate without change of form and do not interact.

At the next order of approximation restrictions on the functions $F_{1}$ and $F_{2}$ are necessary in order to maintain the validity of the expansion procedure; equations governing the slow evolution of these waves as they interact are developed. The restrictions on the functions $F_{1}$ and $F_{2}$ are that

(i) $\quad F_{1}$ and $F_{2}$ are bounded,

$$
\left.\begin{array}{l}
F_{1}^{b} F_{1}(\xi) d \xi \text { and } \int_{a}^{b} F_{2}(\xi) d \xi \text { are bounded for all } a \text { and } b, \\
d F_{1} / d \xi \text { and } d F_{2} / d \xi \text { are bounded. }
\end{array}\right\}
$$

These conditions ensure that the leftward-and rightward-running waves are too weak and too localized to interact with each other at this order of approximation; however, each of the wave sets has a long time to interact with itself and an evolution equation is obtained for each of the wave sets. The dimensional equation for the rightward-running waves is

$$
\eta_{t}+(g h)^{\frac{1}{2}} \eta_{x}+\frac{3}{2}(g h)^{\frac{1}{2}} \eta \eta_{x}+\frac{1}{6} h^{2}(g h)^{\frac{1}{2}} \eta_{x x x}=0 .
$$

More convenient variables for describing the rightward-running waves are

$$
\left.\begin{array}{c}
r=h^{-1}\left[x-(g h)^{\frac{1}{2}} t\right], \quad \tau=\frac{1}{6}(g / h)^{\frac{1}{2}} t, \\
f(r ; \tau)=(3 / 2 h) \eta(x ; t) .
\end{array}\right\}
$$

In terms of these variables ( 7 ) becomes

$$
f_{\tau}+6 f f_{r}+f_{r r r}=0
$$


which is (1); a similar equation can also be found for the set of leftward-running waves. Note that, when the initial wave sets satisfy the conditions on the initial data given by (2) and (3), then a fortior $i$ the conditions (6) are satisfied.

The asymptotic solution of (1) was discussed in part 1 of this study. In the present paper the following features of the asymptotic solution are compared with experimental results.

(i) An arbitrary rightward-running disturbance which initially satisfies (2) and (3) evolves into a finite number of permanent waves (solitons) and a dispersive wave train which oscillates about the still water level.

(ii) When the net volume $V$ per unit width of the disturbance is finite and positive, i.e.,

$$
V=\int_{-\infty}^{\infty} f(r ; 0) d r>0
$$

at least one soliton emerges. Each soliton is a positive permanent wave whose velocity of propagation (relative to $(g h)^{\frac{1}{2}}$ ) is proportional to its amplitude. The maximum amplitude of the leading soliton is $2|\lambda|$, where

$$
\lambda=\min _{\psi(r)}\left\{\int_{-\infty}^{\infty}\left[\left(\frac{d \psi}{d r}\right)^{2}-f(r ; 0) \psi^{2}\right] d r / \int_{-\infty}^{\infty} \psi^{2}(r) d r\right\} .
$$

This amplitude cannot exceed $2 f_{0}$, where $f_{0}=\max _{r} f(r ; 0)$. When the solitons are well separated the local shape of each soliton is given by

$$
f=2|\lambda| \operatorname{sech}^{2}\left\{|\lambda|^{\frac{1}{2}}\left(r-r_{0}-4|\lambda| \tau\right)\right\}
$$

(iii) The number $N$ of solitons that evolve during propagation can be determined from the initial data by counting the negative real eigenvalues of the problem

$$
\left.\begin{array}{c}
d^{2} \psi / d r^{2}+[\lambda+f(r ; 0)] \psi=0 \\
\psi \rightarrow 0 \text { as }|r| \rightarrow \infty
\end{array}\right\}
$$

Simple bounds on $N$ for various initial data were discussed in part 1 . When the initial data vanish outside a finite interval (say $f(r ; 0) \equiv 0$ for $r<r_{1}$ and $r>r_{2}$, where $r_{1}<r_{2}$ ) as is the case for the experiments to be examined in this study, $N$ can be computed exactly by integrating the equation

with

$$
\left.\begin{array}{c}
d^{2} \phi / d r^{2}+f(r ; 0) \phi=0, \\
\phi\left(r_{1}\right)=1, \quad d \phi\left(r_{1}\right) / d r=0,
\end{array}\right\}
$$

for $r>r_{1}$, and counting the zeros of $\phi$. The function has $N$ simple zeros, all at finite $r$.

(iv) When $f(r ; 0) \leqslant 0$ no solitons emerge and the asymptotic solution consists only of the dispersive train of oscillatory waves (tail). After its development this wave train can be described as slowly varying, with the wavenumber increasing from $k=0$ near the leading region of the advancing wave train to higher values towards the rear. The local group velocity of the wave train, relative to $(g h)^{\frac{1}{2}}$, is negative $\left(-3 k^{2}\right)$; hence, the whole tail will travel more slowly than the slowest soliton. The maximum amplitude of the waves in the tail decays algebraically 
as the waves disperse. Unfortunately, the predicted decay rates of the tail (soe, for example, Ablowitz \& Newell 1973) cannot be tested in the following experiments owing to the presence of viscous energy losses.

(v) Two other important classes of initial data which are not described above are

$$
\text { (I) } \int_{-\infty}^{\infty} f(r ; 0) d r<0, \quad f(r ; 0) \$ 0 \text { for all } r
$$

and

(II) $\int_{-\infty}^{\infty} f(r ; 0)=0$

i.e. in case $I$ the net volume per unit width in the initial data is negative but positive waves are present and in case II the net volume per unit width in the initial data is zero. Experimental evidence will be presented for these cases which indicates that the evolution of solitons depends on the detailed structure of the initial data. (Recall that the existence of solitons is guaranteed when

$$
\int_{-\infty}^{\infty} f(r ; 0) d r>0
$$

regardless of the exact structure of $f(r ; 0)$ : only the number and amplitudes of the solitons depend on $f(r ; 0)$.)

(vi) After a sufficiently long time, the solitons and the oscillatory tail separate. Then the solitons may continue to interact among themselves, but the entire wave appears as a linear superposition of the tail and the set of solitons, even though (1) is nonlinear.

(vii) The horizontal position of the centroid of a wave system in the travelling co-ordinate system is given by

$$
\chi=\int_{-\infty}^{\infty} r f(r ; \tau) d r / \int_{-\infty}^{\infty} f(r ; \tau) d r
$$

Benjamin \& Mahony (1971) have shown for the complete water-wave problem ( $\epsilon$ arbitrary) that the velocity $\partial \chi / \partial \tau$ of the centroid remains constant during propagation in the absence of viscous stresses. Using the following quantities which are conserved by the KdV equation (see Miura, Gardner \& Kruskal 1968),

$$
\int_{-\infty}^{\infty} f d r, \quad \int_{-\infty}^{\infty} f^{2} d r, \quad \int_{-\infty}^{\infty}\left(r f-3 \tau f^{2}\right) d r
$$

it follows that the constant velocity of the centroid is given by

$$
\frac{\partial \chi}{\partial \tau}=3 \int_{-\infty}^{\infty} f^{2} d r / \int_{-\infty}^{\infty} f d r
$$

Hence, the centroid velocity (relative to $(g h)^{\frac{1}{2}}$ ) is positive or negative depending on whether the net volume per unit width of the initial wave is positive or negative. 


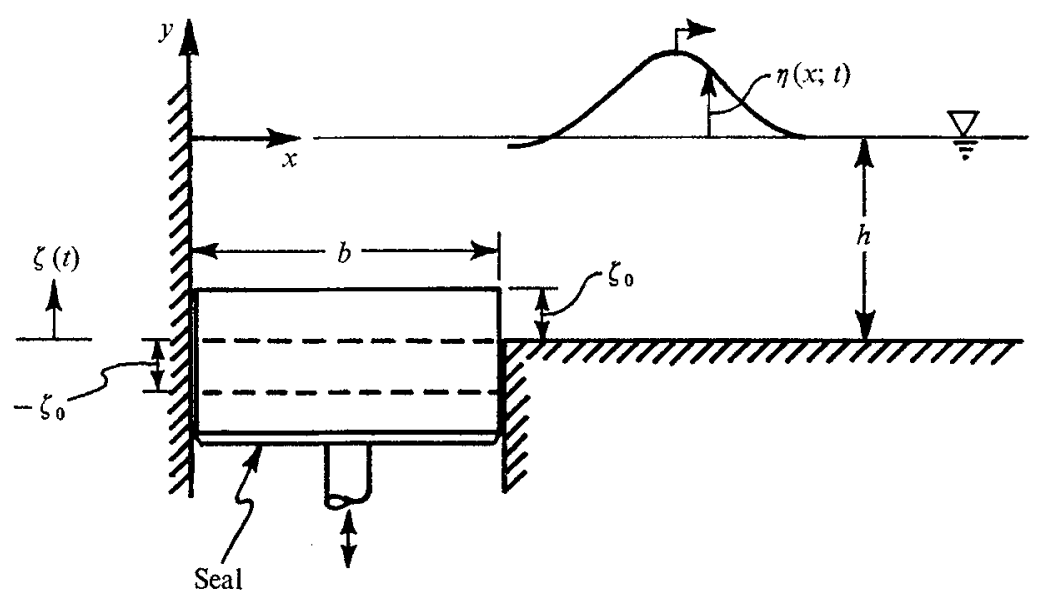

Figure 2. Schematic drawing of the wave generator.

\section{Wave generation and experimental equipment}

A series of laboratory experiments was conducted using a wave tank $103.8 \mathrm{ft}$ $(31.6 \mathrm{~m})$ long, $2 \mathrm{ft}(61 \mathrm{~cm})$ deep and $15 \frac{1}{2} \mathrm{in} .(39.4 \mathrm{~cm})$ wide; the vertical side walls of the tank were constructed of glass throughout. As shown schematically in figure 2 , the wave generator consisted of a rectangular piston located in the tank bed adjacent to the upstream end wall of the wave tank which could be displaced vertically either up or down. The piston spanned the tank width and had a length $b$ in the direction of wave propagation which could be varied. For the experiments discussed herein, lengths $b=61 \mathrm{~cm}$ and $b=30.5 \mathrm{~cm}$ were used. The time-displacement history of the piston, denoted by $\zeta(t)$, was prescribed and controlled by an electro-hydraulic-servo system (cf. Hammack 1972 or 1973). A rubber seal was attached around the bottom edge of the piston in order to confine the fluid domain to the region above the wave maker.

For many of the experiments discussed herein, the piston moved monotonically from its initial position (level with the fixed bed of the wave tank) to a final elevation $\zeta_{0}$ (see figure 2). The fluid was quiescent prior to the bed motion; hence, the net volume $V$ per unit width in the generated wave system was $b \zeta_{0}$. The detailed time-displacement histories of the bed motion will not be discussed since this study is primarily concerned with the propagation of the waves rather than their generation. (Typical bed motions used have been described by Hammack (1973).) In addition to the monotonic bed motions, experiments were conducted in which the piston oscillated through one or more cycles before stopping either at the initial bed position $(V=0)$ or at a position of permanent displacement $(V \neq 0)$. These experiments were used to investigate the asymptotic behaviour of initial wave data satisfying (14) or (15). It should be noted that the upstream end wall of the wave tank adjacent to the wave maker is a plane of symmetry for these wave motions. Hence, the length $b$ actually represents the half-length of a hypothetical piston occupying the region $-b<x<b$, the waves propagating 
out of the generation region in the direction of increasing $x$ being the rightwardrunning set that would be generated by a piston symmetric about $x=0$ with a length $2 b$.

Wave measurements were made during each experiment at four positions using parallel-wire resistance gauges and an oscillograph recorder. In the experiments to be discussed the undisturbed fluid depth was $h=5 \mathrm{~cm}$, and waves were measured at $(x-b) / h=0,(x-b) / h=20,(x-b) / h=180$ or 200 and at $(x-b) / h=400$. It should be emphasized that this method of wave measurement yielded a temporal record of the water surface displacement at a fixed position $x_{0}$ rather than a spatial description of the wave structure at a fixed time $t_{0}$, which is required for a direct comparison with the theory presented in $\$ 2$. (Quantitative spatial measurements of waves at a fixed time are difficult to perform accurately for the long waves of moderate amplitude considered in this study.) However, the temporal wave structure measured at a fixed position $x_{0}$ should differ from the spatial wave structure measured at a fixed time $t_{0}=x_{0} /(g h)^{\frac{1}{2}}$ by quantities $O(\epsilon)$, i.e. no significant evolution of the wave occurs during a temporal measurement whose duration is small compared with $(h / g)^{\frac{1}{2}} / \epsilon$. This follows from the assumptions, stated in $\$ 2$, required to derive (1) as a model for this problem. Thus, the choice of measurements in the experimental programme limits the range of $\epsilon$ for which comparisons with theory are meaningful.

It should also be noted that an essential requirement for relating these temporal measurements to spatial wave profiles is that the recorded surface motions be the result of the passage of a free wave as implied in $\S 2$. This requirement is not satisfied by measurements in the fluid domain directly above the piston $(x<b)$, where changing elevations of the water surface are a combination of both free and forced wave motions. For this reason, wave records identified herein as being recorded at $(x-b) / h=0$ were actually made slightly downstream of the piston.

\section{Experimental results}

As indicated in $\S 1$, the purpose of this study is to compare experimental and theoretical results for wave evolution over a time scale sufficiently large for the asymptotic features of the wave system to develop. An important concept in the use of experimental data for this comparison is that of a sorting time $t_{s}$, which is defined as the time required for a set of ordered solitons, if present, to evolve from the initial data. The time for this evolution to occur can be estimated by considering for simplicity an initially positive and rightward-running wave satisfying (3) with a maximum amplitude $\eta_{0}$ and an effective length $l$. The amplitude of the largest soliton which can evolve from these initial data cannot exceed $2 \eta_{0}$; hence, an upper bound for the dimensional speed of this leading soliton is $(g h)^{\frac{1}{2}}\left(1+\eta_{0} / h\right)$. A lower bound for the speed of the smallest soliton evolving from these initial data is $(g h)^{\frac{1}{2}}$; hence, these two solitons move relative to each other (ignoring phase shifts) with a maximum speed $s=(g h)^{\frac{1}{2}}\left(\eta_{0} / h\right)$. The initial data are expected to sort themselves into an ordered set of solitons in the time $t_{s}$ required for the largest and smallest solitons to travel a distance $l$ relative 
to each other; hence, the approximate magnitude of the sorting time is given by

$$
t_{s}=l / s=(h / g)^{\frac{1}{2}}\left(\eta_{0} / l\right)^{-1} \quad \text { or } \quad t_{s}(g / h)^{\frac{1}{2}}=O\left(\epsilon^{-\frac{3}{2}}\right) .
$$

Since the sorting time given by (18) has been obtained by ignoring phase shifts, it should be most applicable when the number of solitons evolving from the initial data is small, which is the case for the experiments discussed herein. (It should also be noted that the sorting time given by (18) is implied by Whitham's (1965) method of averaging. To see this consider as initial data for (1) a slowly varying wave train whose amplitude varies from a maximum $\eta_{0}$ to zero over an effective length $l$. The small parameter $\delta$ required for Whitham's theory is the spatial rate of modulation of the amplitude, i.e. $\delta \sim \eta_{0} / l \ll 1$. The theory then describes the evolution of such a wave train for a time $O\left(\delta^{-1}\right)$, which is consistent with (18).) It is more convenient in the present study to use a sorting distance $d_{\mathrm{s}}$ to determine the approximate distance required for solitons to evolve from the initial data. This distance is given by

$$
d_{s}=(g h)^{\frac{1}{2}} t_{s} \quad \text { or } \quad d_{s} / h=\left(\eta_{0} / l\right)^{-1}=O\left(\epsilon^{-\frac{3}{2}}\right) .
$$

It should be emphasized that this calculation is only expected to provide an order-of-magnitude accuracy for the sorting distance. In the experiments which follow, a sorting distance has been calculated for each set of initial data in order to determine whether the available test section of the wave tank is sufficiently long for the asymptotic features of the solution to be distinguished. In the computation of the sorting distance the length $l$ has been chosen as the interval over which the initial data are non-zero while $\eta_{0}$ is taken as the absolute value of the maximum amplitude which occurs in the initial data. Experiments for which $d_{s}$ is much greater than the effective tank length have been omitted from consideration.

$$
\text { 4.1. Initial data satisfying } \int_{-\infty}^{\infty} f(r ; 0) d r>0
$$

Four experiments are presented in order to examine the asymptotic features of wave evolution when the net volume $V$ per unit width of the initial wave is positive. Figure 3 shows a series of measured wave profiles resulting from a monotonic uplift of the piston to an elevation $\zeta_{0}=+0.5 \mathrm{~cm}$ in a water depth of $5 \mathrm{~cm}$. Since the length $b$ of the piston was $61 \mathrm{~cm}$ in this experiment, the volume in the generated wave system was $V=30.5 \mathrm{~cm}^{3} / \mathrm{cm}$ (henceforth, values of the experimental parameters $h, b$ and $V$ will be noted in the figure captions only). The normalized wave amplitude $\eta / h$ (or $\frac{2}{3} f$ ) is shown in figure 3 as a function of the non-dimensional time $t(g / h)^{\frac{1}{2}}-(x-b) / h$ (or $r_{0}-r$, where $r_{0}=b / h$ ). The wave traces are presented in this manner to emphasize that they are in fact temporal measurements at a fixed spatial location which are subject to an error $O(\epsilon)$ when interpreted as spatial records at a fixed time (see §3). (Unfortunately, a rightward-running wave system necessarily appears to be running to the left when presented in this manner since the spatial co-ordinate $(x$ or $r)$ increases to the left. Hence, the leading portion of an advancing wave train will always appear to the left in the figures.) 


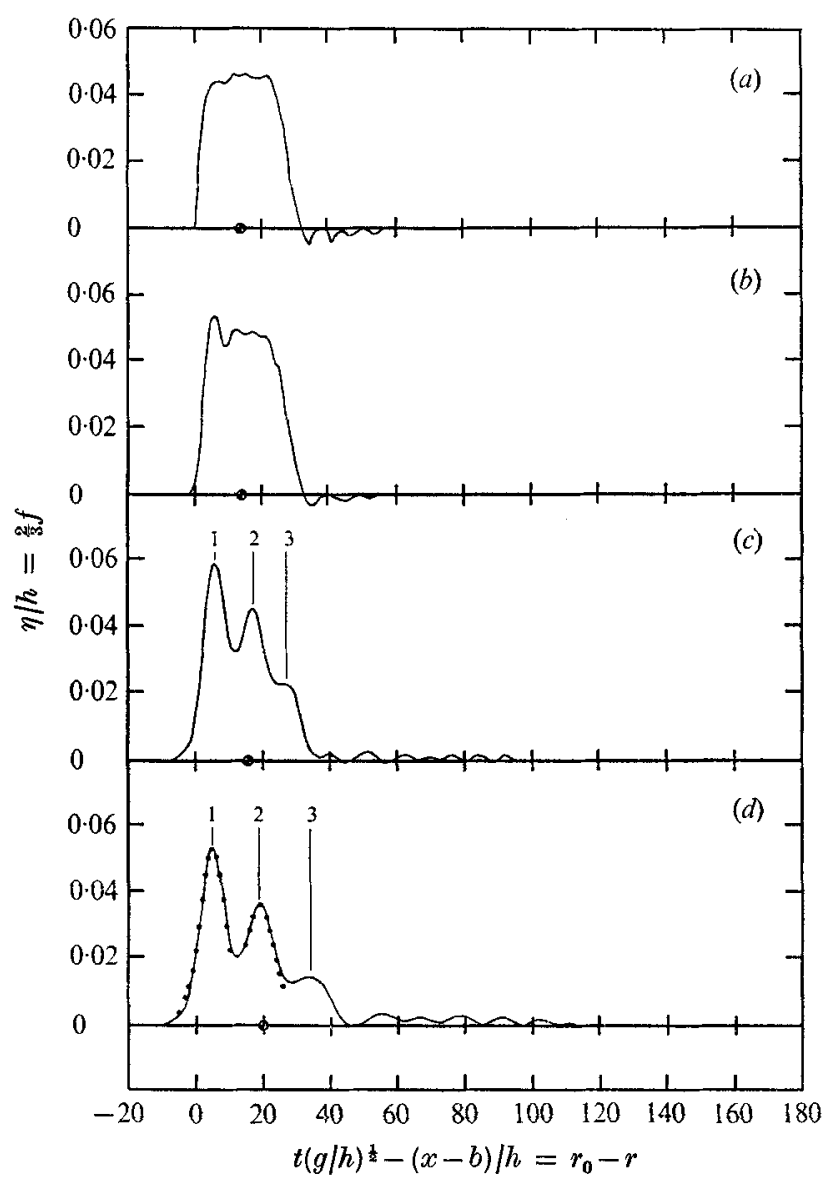

Figure 3. Experimental wave systems: $h=5 \mathrm{~cm}, b=61 \mathrm{~cm}, V=30.5 \mathrm{~cm}^{3} / \mathrm{cm}, N=3$, $d_{s} / h=700 .-$ measured profiles; $\bigcirc$, soliton profiles computed using $(11) ; \boldsymbol{\Theta}$, location of centroid. $(a)(x-b) / h=0 .(b)(x-b) / h=20$. $(c)(x-b) / h=180$. (d) $(x-b) / h=400$.

The initial wave at $(x-b) / h=0$ in figure 3 is approximately a square wave (similar to the permanent deformation of the bed in the generation region) whose amplitude is positive. Based on the maximum amplitude and the length of these initial data (ignoring the small oscillations trailing the primary wave) the sorting distance is found to be approximately 700 water depths. Since only 400 water depths are available for observation in the test section of the wave tank, the asymptotic features of the evolving wave train are not expected to develop fully during observation. However, as will be shown, the asymptotic features can be distinguished with sufficient accuracy to warrant examination.

The wave system in figure 3 appears to sort itself into three positive waves (solitons), which are identified by separate crests (local maxima) and are labelled 1, 2 and 3 in the wave traces at the last two stations of measurement. These three waves are ordered by amplitude and are followed by a small amplitude train of oscillatory waves which spreads to the right during propagation; hence, the group velocity of these oscillatory waves is negative relative to $(g h)^{\frac{1}{2}}$. (In the 
co-ordinate system used, a point moves to the left or right in succeeding measurements depending on whether its velocity is greater than or less than $(g h)^{\frac{1}{2}}$, respectively.) During propagation between the last two stations of measurement, the three primary waves separate with the largest wave, wave 1 , travelling at a velocity slightly greater than $(g h)^{\frac{1}{2}}$. In order to examine the shape of the evolving waves, profiles of two solitons as defined by (11) have been superposed on the two leading waves at the last station of observation in figure 3 . These exact profiles have been computed from (11) using the maximum amplitude of the measured waves to determine the magnitude of $|\lambda|$, which appears in this equation. The exact profiles are not extended into the regions of the two leading waves where interaction with surrounding wave structure is occurring. Comparison of the theoretical profiles with the measured waves clearly indicates that the two leading waves have attained the shape of solitons near their crests. (A similar statement cannot be conclusively made for wave 3 , which is still strongly interacting with the surrounding waves at the last station of observation.) Further evidence that the three primary waves at the last station of observation are indeed solitons is provided by numerically integrating (13) using the wave profile at $(x-b) / h=0$ as the potential $f(r ; 0)$. Computation of the total number of solitons expected to evolve yields $N=3$, which agrees with the observed pattern of evolution.

Three features of the observed evolution in figure 3 are not in general agreement with the theoretical model; each of these features can be attributed to viscous effects in the experiments. First, the observed speeds of the last two solitons do not exceed $(g h)^{\frac{1}{2}}$. Second, the position of the centroid (computed by numerically integrating the data on each wave record at successive stations of measurement) indicates that the centroid velocity is not constant and is less than $(g h)^{\frac{1}{2}}$. Since the net wave volume for these initial data is positive, the centroid velocity should exceed $(g h)^{\frac{1}{2}}$. These discrepancies in centroid and soliton propagation speeds are probably due to the presence of viscous stresses around the wetted perimeter of the wave-tank cross-section. The third feature of observed wave evolution which differs from the theoretical model is the reduction in soliton amplitude after separation and during propagation. This phenomenon will be discussed in more detail in $\$ 4.4$ and is a result of energy losses in the viscous boundary layers which develop around the boundaries of the fluid domain during wave propagation. Energy losses as well as boundary stresses are not modelled by the $\mathrm{KdV}$ equation.

A second example of wave evolution resulting from a monotonic uplift of the bed in the generation region is shown in figure 4 . The evolution of this particular wave system is interesting since the initial data at $(x-b) / h=0$ are closely approximated over most of the profile by

$$
f=\frac{3}{2} \alpha \operatorname{sech}^{2} \beta\left(r_{0}-r\right),
$$

where $r_{0}-r=t(g / h)^{\frac{1}{2}}$ since $(x-b) / h=0, f=\frac{3}{2} \eta / h, \alpha=0.082$ and $\beta=0.069$. (The profile (20) is shown superposed on the measured initial data in figure 4.) The wave profile given by (20) is of the same form as the soliton solution given by (11) except that the wave amplitude $\frac{3}{2} \alpha$ and length $\beta^{-1}$ are improperly related, i.e. 


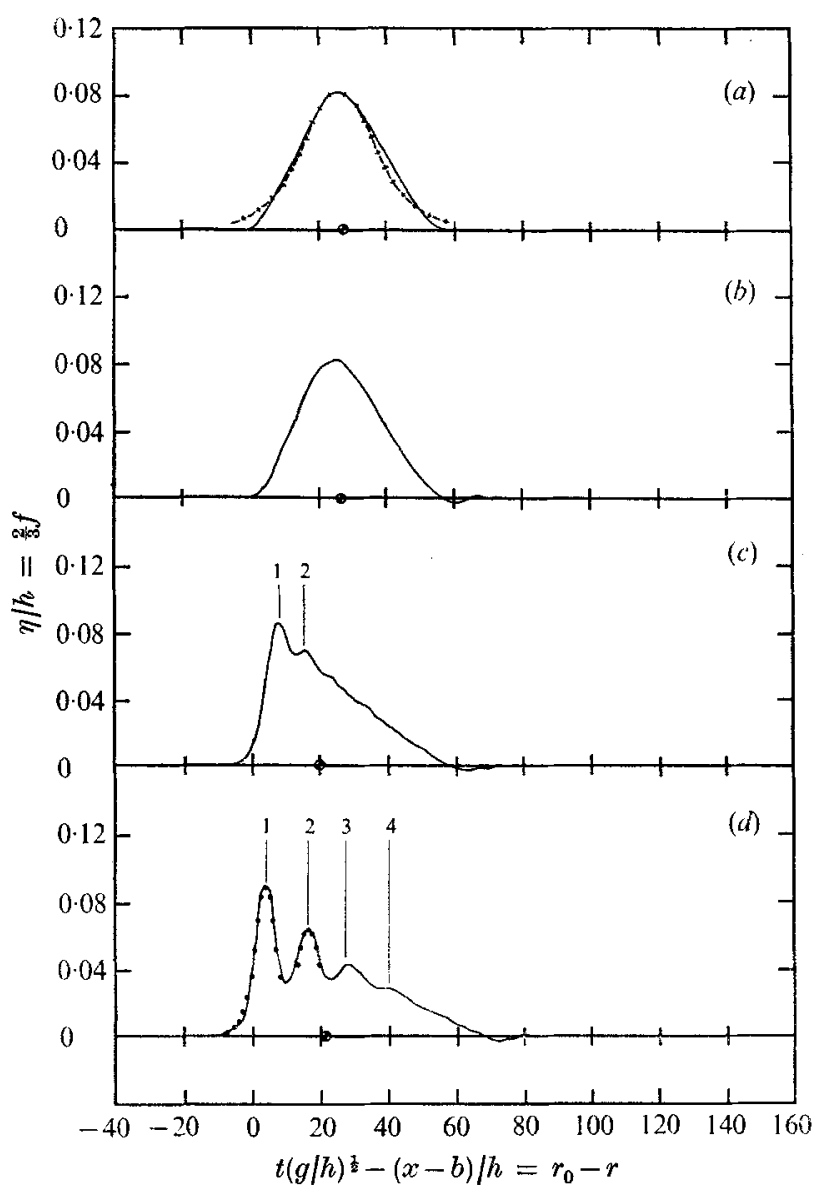

FrguRe 4. Experimental wave systems: $h=5 \mathrm{~cm}, b=61 \mathrm{~cm}, V=61 \mathrm{~cm}^{3} / \mathrm{cm}, N=4$, $d_{s} / h=680$. - measured profiles; $\bullet$, soliton profiles computed using (11); -+- , $f=0.12 \operatorname{sech}^{2}\left\{0.069\left(r_{0}-r\right)\right\} ; \vartheta$, location of centroid. $(a)(x-b) / h=0 .(b)(x-b) / h=20$. (c) $(x-b) / h=180 .(d)(x-b) / h=400$.

$\beta^{2} \neq \frac{3}{4} \alpha$. During propagation the initial wave evolves away from this $\operatorname{sech}^{2}$ profile. After 180 depths of propagation two local maxima (crests) are distinguishable and are labelled waves 1 and 2 . At the last station of observation four local maxima are observed; the leading two waves clearly resemble solitons near their crests as indicated by comparison with exact soliton profiles computed from (11) which are shown superposed on the experimental measurements at this station. (Equation (11) has again been plotted using the maximum amplitude of the measured waves to determine the magnitude of $|\lambda|$; profiles are not extended into regions of interaction of the two waves.) The sorting distance for the initial wave in figure 4 is about 680 water depths; hence, the solitons in the initial data are expected still to be localized at $(x-b) / h=400$ and all of the solitons may not be identifiable. Computing the number of expected solitons by numerically integrating (13) using the initial data of figure 4 as the potential yields $N=4$, which does agree with the observed behaviour at the last station of observation. 


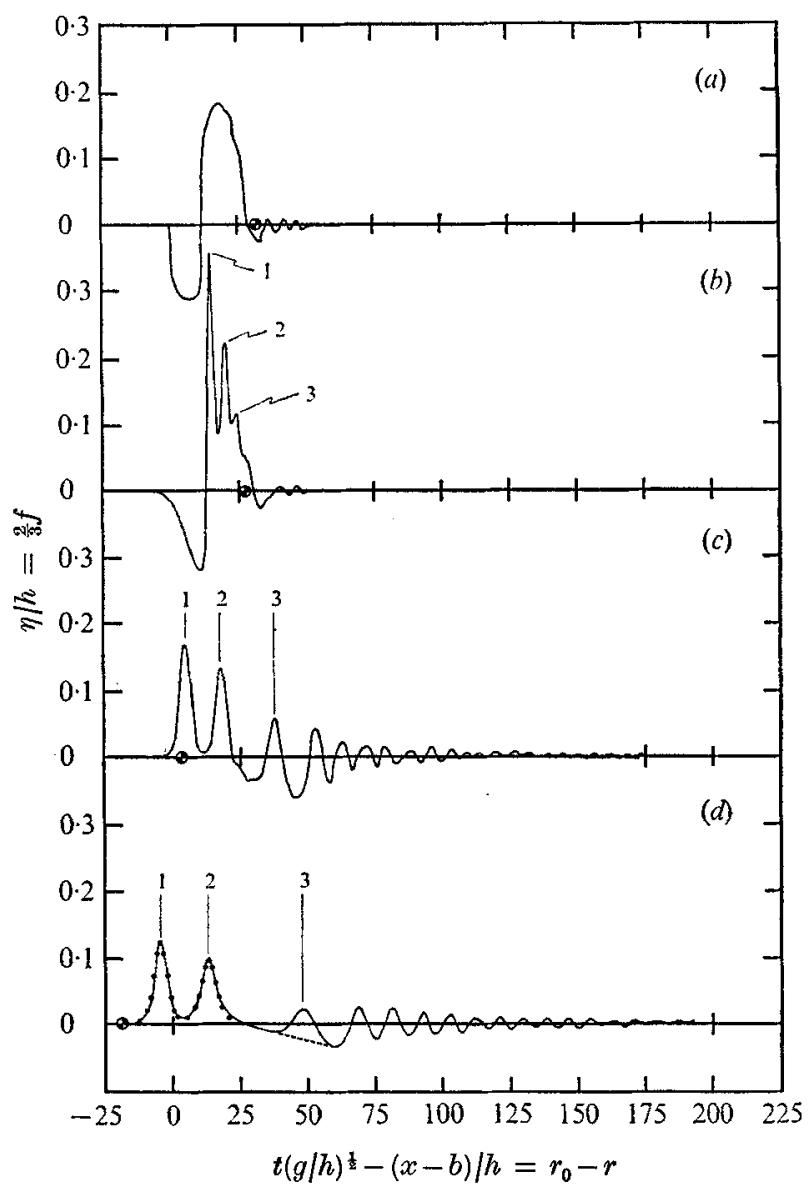

Figure 5. Experimental wave systems: $h=5 \mathrm{~cm}, b=30.5 \mathrm{~cm}, V=30.5 \mathrm{~cm}^{3} / \mathrm{cm}, N=3$, $d_{s} / h=260$. -, measured profiles; $O$, soliton profiles computed using (11); - - - , hypothetical position of the leading portion of the tail; $\theta$, location of centroid. $(a)(x-b) / h=0$. (b) $(x-b) / h=20$. (c) $(x-b) / h=200 .(d)(x-b) / h=400$.

Note that the velocity of the centroid shown in figure 4 is greater than $(g h)^{\frac{1}{2}}$ between the first and third stations of measurement but is slightly less than $(g h)^{\frac{1}{2}}$ between the last two stations.

In order to examine the sufficiency of the requirement that the net wave volume be positive for solitons to evolve, an oscillatory bed motion was used to generate an initial wave system more complicated than those presented in figures 3 and 4 . The resulting waves are shown in figure 5. The initial data at $(x-b) / h=0$ consist of a negative wave followed by a larger positive wave. After only twenty depths of propagation the large positive wave has separated into three separate crests, which have been labelled. The leading negative wave at $(x-b) / h=20$ has essentially remained the same except for a decrease in slope and a stretching of the front face of this wave. (It is noteworthy that the amplitude of wave 1 after 20 depths of propagation is approximately twice that of the positive 


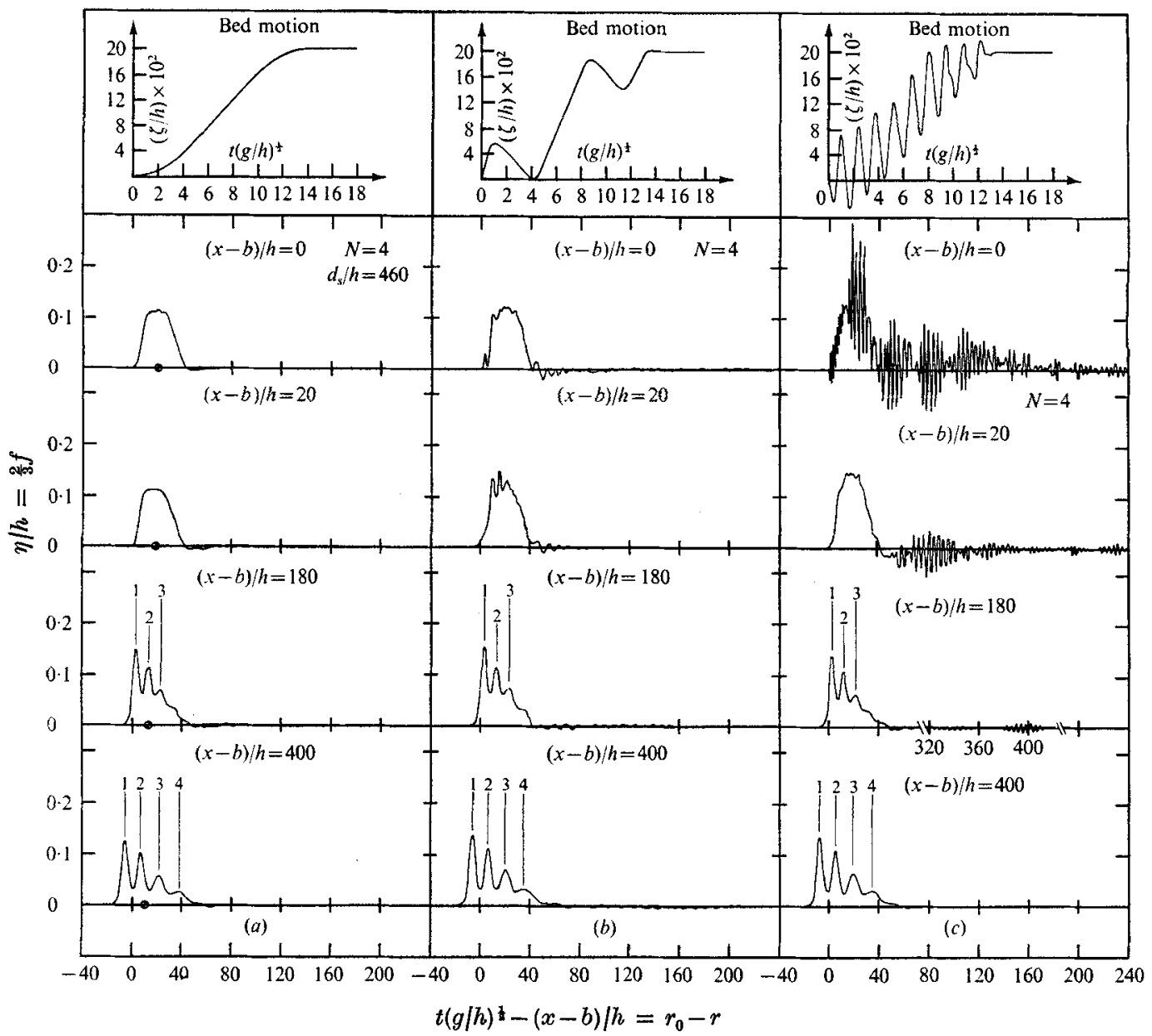

Figure 6. Time-displacement histories of the wave generator and the resulting wave systems: $h=5 \mathrm{~cm}, b=61 \mathrm{~cm}, V=61 \mathrm{~cm}^{3} / \mathrm{cm}$. (a) Mean motion; (b), (c) mean motion with superposed oscillation. $\bigcirc$, location of centroid.

wave in the initial data.) During further propagation the three labelled waves appear to retain their integrity as they progress through the leading negative wave and emerge at the front of the wave train. At the last station of observation, waves 1 and 2 clearly resemble solitons near their crests as indicated by the comparison with exact profiles computed from (11) as previously described and superposed on the measured profile. The third wave is still interacting with the once-leading negative wave, and cannot be unmistakably identified as a soliton. The centroid velocity of this wave system does exceed $(g h)^{\frac{1}{2}}$ throughout the range of observation; however, the velocity is not constant and decreases slightly during propagation.

There has been some controversy in recent literature (Benjamin, Bona \& Mahony 1972) as to whether the high frequency content that would exist in most realistic initial data could invalidate the $\mathrm{KdV}$ equation, which is derived as a model of the propagation of long waves. Consequently, experiments were 
conducted in which an oscillatory motion of the piston ('dither') was superposed on a monotonic mean motion to determine the effect of short waves in the initial data on the asymptotic wave behaviour. The amplitude and frequency of the superposed dither were varied relative to the amplitude and period of the mean motion. Resulting waves from three experiments are presented in figures $6(a),(b)$ and $(c)$ along with the time-displacement history $\zeta(t)$ of the bed motion. Figure $6(a)$ shows the waves resulting from the monotonic mean motion alone. The initial wave consists essentially of a positive mound of water, which evolves into four separate waves after 400 depths of propagation. Computation of $N$ from (13) using these initial wave data as the potential suggests that four solitons should evolve; a sorting distance of approximately 460 water depths is found for these initial data.

Figure $6(b)$ shows the resulting waves when a dither with an amplitude and period half those of the mean motion of figure $6(a)$ is superposed on the mean motion. The initial wave in figure $6(b)$ contains oscillatory waves whose period and amplitude are proportional to those of the dither. These high frequency waves (relative to the frequency of the mean wave) are still evident after twenty depths of propagation. At the third station of observation the oscillatory waves trail behind the evolving solitons, whose structure is almost identical with that of the wave system in figure $6(a)$ at the equivalent measurement position. After 400 depths of propagation four solitons are observed in figure $6(b)$ with amplitudes and spacing identical to the results in figure $6(a)$; hence, the higher frequency waves in the initial data do not appear to have any permanent effect on the evolving solitons in this experiment. Further evidence of this insensitivity of the solitons to high frequency content of the initial data is also indicated by the computation of $N$ for the initial data in figure $6(b)$; again four solitons are calculated to evolve asymptotically.

The waves resulting when the period of the superposed dither is reduced to one tenth of the period of the mean motion while the relative amplitude of the dither is maintained at one half are presented in figure $6(c)$. This bed motion generated a system of cross-waves (waves propagating laterally across the tank) in the generation region, which were recorded by the transducer at $(x-b) / h=0$. The large amplitude oscillations in the measured profile at $(x-b) / h=0$ which occur after the bed motion has ceased are cross-waves; hence, this wave system contains spurious information if used in the comparison with the theory for onedimensional (longitudinal) wave propagation presented in $\S 2$. The recorded wave structure at $(x-b) / h=20$ does consist almost entirely of longitudinally propagating waves and can be considered as the initial data for the evolving waves at the remaining downstream positions. This initial wave system consists of a leading positive wave (apparently generated by the mean motion of the wave maker) followed by a dispersive train of high frequency waves which were generated by the oscillations of the wave maker as well as the longitudinal pressure gradients created by the cross-waves. During further propagation these dispersive waves are left behind by the evolving mean wave (note that the abscissa has been broken at $(x-b) / h=180$ in order to show the largest trailing wave group). After 400 depths of propagation the evolving wave structure is almost 


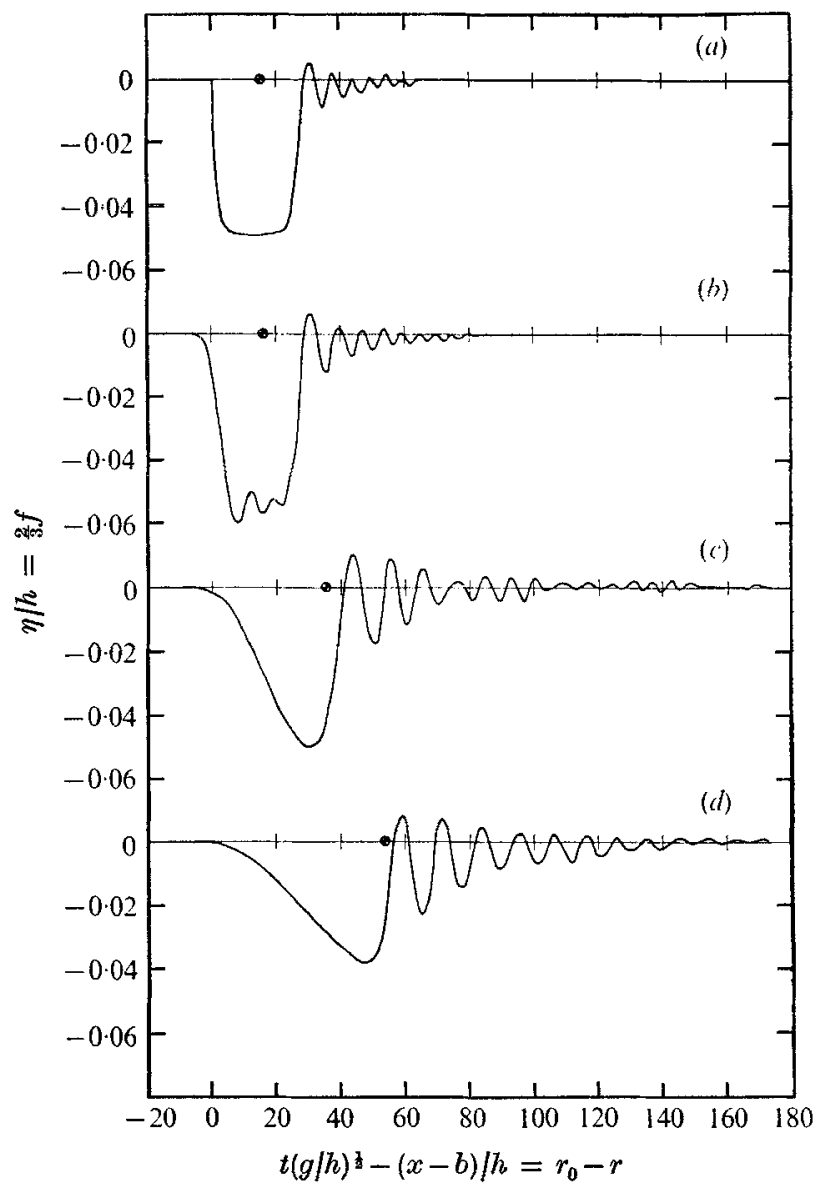

FrGURe 7. Experimental wave systems: $h=5 \mathrm{~cm}, b=61 \mathrm{~cm}, V=-30.5 \mathrm{~cm}^{3} / \mathrm{cm}, N=0$, $d_{s} / h=600$. - measured profiles; - , location of centroid. $(a)(x-b) / h=0$. (b) $(x-b) / h=20$. (c) $(x-b) / h=180 .(d)(x-b) / h=400$.

identical to that observed in figures $6(a)$ and $(b)$; the dispersive wave train has decayed to such an extent at this position (as a combined result of viscous effects and frequency separation) that it is no longer measurable. Using the initial wave system at $(x-b) / h=20$ in figure $6(c)$ as the potential in (13), four solitons are again computed to evolve asymptotically just as observed. Hence, it appears that high frequency (or wavenumber) content in the initial data does not have any significant effect on the evolving solitons.

$$
\text { 4.2. Initial data satisfying } \int_{-\infty}^{\infty} f(r ; 0) d r<0
$$

In order to demonstrate the primary features of the asymptotic solution when the net volume in the initial wave system is negative, results from two experiments are presented. Figure 7 shows the waves resulting from a monotonic downthrow of the piston. The initial wave at $(x-b) / h=0$ is approximately a negative 
square wave; the sorting disturbance for these initial data is approximately 600 water depths.

No positive waves resembling solitons appear to develop in figure 7 during 400 depths of propagation. Even though the sorting distance does exceed the range of observation, the results presented in $\S 4.1$ would appear to indicate that, if any solitons are present, their crests should be distinguishable at $(x-b) / h=400$ as they progress through the leading negative wave. The wave train that does develop from these initial data consists of a leading negative wave whose frontal slope decreases and elongates with time. It is in this region of the wave train that the local wavenumber approaches zero with inoreasing time as the characteristic wavelength, $l \propto k^{-1}$, grows (see Hammack (1973) for a detailed discussion of this behaviour). The increasing wavelength in this region also decreases the importance of frequency dispersion and nonlinear effects dominate the wave behaviour. Following a period of initial growth, the amplitude of the leading wave decays during further propagation. The leading negative wave is followed by a dispersive train of oscillatory waves whose amplitude and wavenumber are slowly modulated ( $k$ increasing toward the rear of the train). The group velocity of these oscillatory waves is less than $(g h)^{\frac{1}{2}}$, and the waves spread behind the leading wave. This description of the evolving wave train in figure 7 agrees with the theoretical description of the dispersive train corresponding to the continuous spectrum presented in $\$ 2$.

It is suggested that the dispersive wave train evolving in figure 7 is characteristic for any initial data which satisfy (2) and (3) and have a non-zero contribution from the continuous spectrum. Returning briefly to figure 5 , the sets of waves recorded at the last two stations may each be viewed as a set of three wellseparated solitons, superposed on an oscillatory tail which is similar to that in figure 7. In these wave records, the first two solitons have separated from the tail, while the third soliton is distinguishable and is still climbing the front slope of the negative wave. The hypothetical position of this front slope on which it is suggested that the third soliton is climbing is indicated in figure 5. This superposition of a soliton on the front face of the tail can also be seen in figures 9 and 10 . Hence, it appears that the evolution of the solitons and the tail do become independent, as the theory suggests.

Further evidence relating to the suggested pattern of evolution for complicated waves in the initial data is presented in figure 8 , where the initial wave system consists of a positive wave followed by a slightly larger negative wave $(V<0)$. In this case the leading positive wave appears to evolve into two positive waves while the negative wave evolves in the same manner as presented in figure 7. Computations using these initial data as the potential in (13) indicate that one soliton should develop asymptotically; hence, it is anticipated that wave 2 would eventually disappear, as it appears to be doing between the last two stations of observation. It should be noted that the wave system at the last station of observation in figure 8 is so long that the entire train could not be measured before the reflected wave from the end of the wave tank returned to the point of measurement; hence, no centroid position could be determined at this station. 


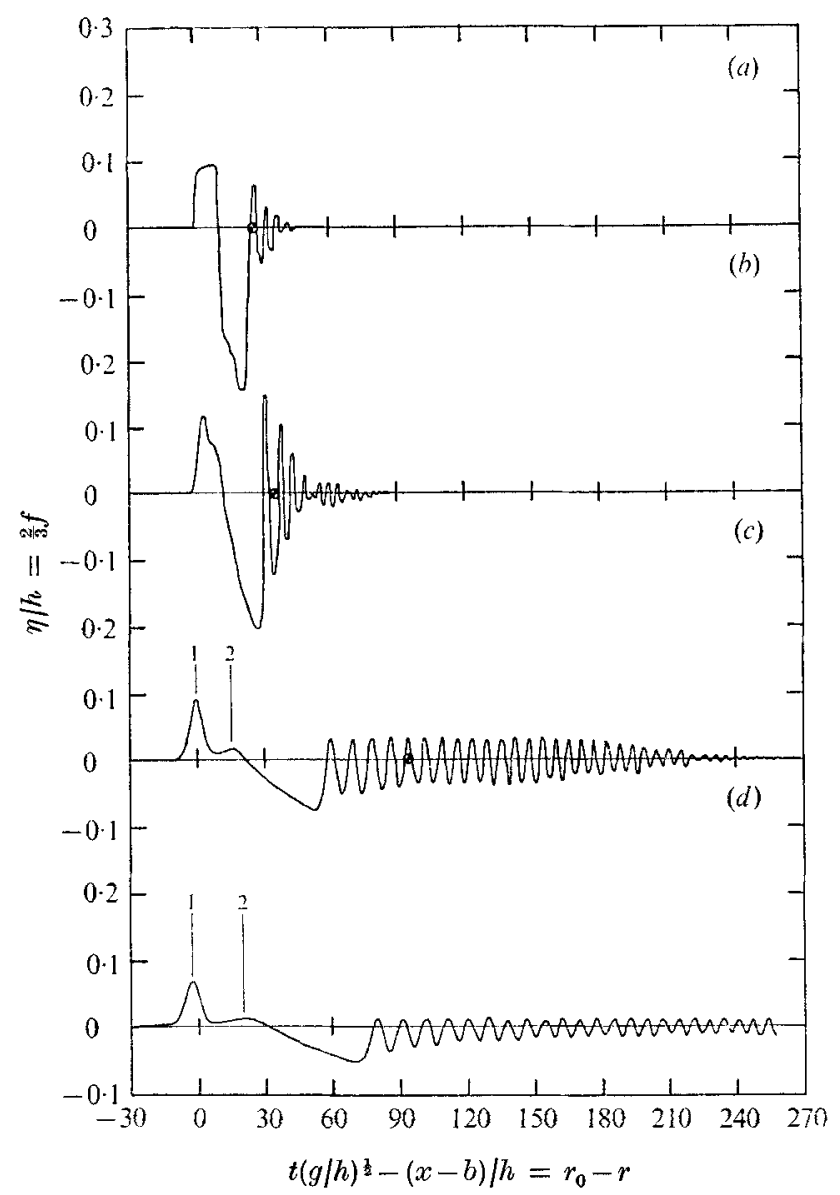

Figure 8. Experimental wave systems: $h=5 \mathrm{~cm}, b=30.5 \mathrm{~cm}, V=-30.5 \mathrm{~cm}^{3} / \mathrm{cm}$, $N=1, d_{s} / h=110$. - measured profiles; $\boldsymbol{\theta}$, location of centroid. $(a)(x-b) / h=0$. (b) $(x-b) / h=20 .(c)(x-b) / h=200 .(d)(x-b) / h=400$.

$$
\text { 4.3. Initial data satisfying } \int_{-\infty}^{\infty} f(r ; 0) d r=0
$$

The theory presented in part 1 of this paper provided no insight into the asymptotic wave behaviour when the net volume in the initial data is zero. Two experiments have been conducted in order to investigate the asymptotic wave behaviour for two particular initial systems satisfying (15). Figure 9 shows the waves resulting when the wave maker is oscillated through one cycle about its initial position. Theinitial data essentially consist of two positive wavesseparated by a single negative wave. Application of (13) to these initial data indicate that three solitons should evolve. After 200 depths of propagation the leading positive wave in the initial data has evolved into one identifiable soliton (labelled 1) as indicated by a comparison with an exact soliton shape computed from (11) for a soliton whose maximum amplitude equals the amplitude of the measured wave. The frontal slope of the negative wave in the initial data has decreased during 


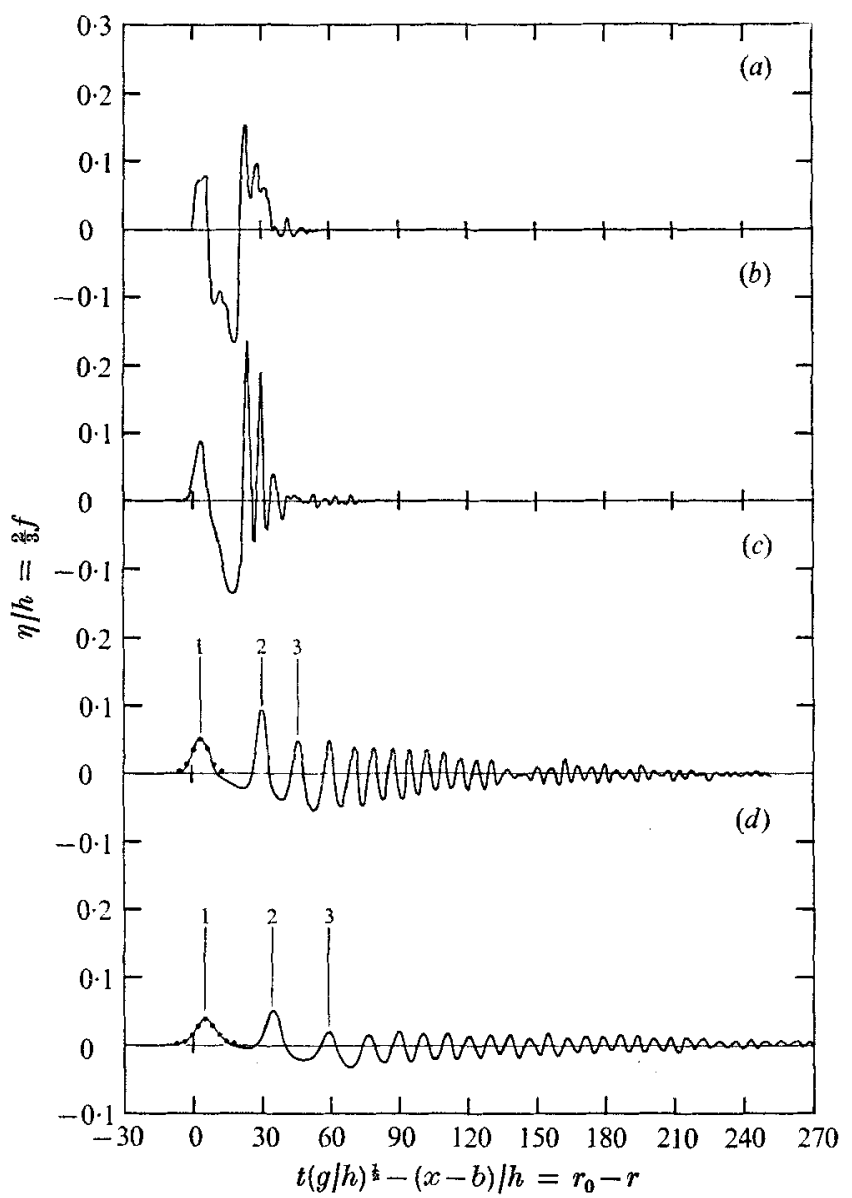

FigURe 9. Experimental wave systems: $h=5 \mathrm{~cm}, b=30.5 \mathrm{~cm}, V=0, N=3, d_{s} / h=220$. - measured profiles; $\bullet$, soliton profiles computed using (11). (a) $(x-b) / h=0$. (b) $(x-b) / h=20 .(c)(x-b) / h=200 .(d)(x-b) / h=400$.

propagation while two positive waves (labelled 2 and 3 ) appear to evolve from the trailing positive wave in the initial data. These latter two waves appear to be propagating along the front face of the evolving negative wave. This pattern of evolution continues through the last station of observation, where wave 2 has almost reached the front of the negative wave system. It does appear that the amplitude of wave 2 is greater than that of wave 1 ; hence, the second wave would be expected to overtake, pass through and emerge in front of wave 1 during further propagation. The tail behind these solitons is well developed at the last station of measurement and is spreading behind the solitons with time. (No centroid position is computed for these wave systems since the net volume is zero.)

Figure 10 shows the evolution of a second initial wave system for which the net volume is zero; this system consists of two primary negative waves separated by a larger positive wave. Application of (13) to these initial data suggests that two solitons should evolve. The large positive wave in the initial data separates into two positive waves after only twenty depths of propagation (neglecting the local 


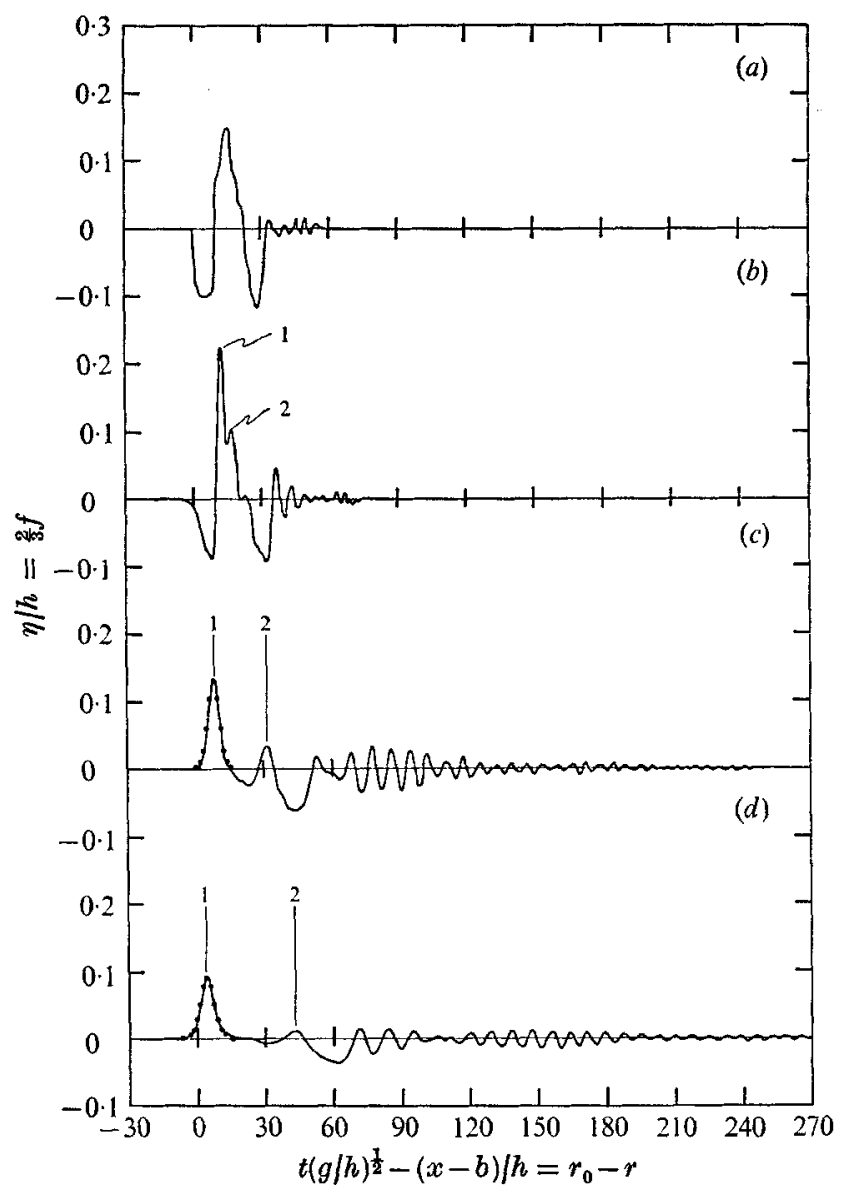

FIgURe 10. Experimental wave systems: $h=5 \mathrm{~cm}, b=30.5 \mathrm{~cm}, V=0, N=2$, $d_{s} / h=230$. - , measured profiles; $O$, soliton profiles computed using (11). $(a)(x-b) / h=0$. (b) $(x-b) / h=20$. (c) $(x-b) / h=200$. (d) $(x-b) / h=400$.

maximum that occurs at the still-water level). At the third station of observation, wave 1 has already progressed to the front of the evolving wave train and clearly has the shape of a soliton as indicated by an exact soliton profile shown superposed on the measured wave. The second labelled wave appears to be progressing along a front slope of a negative wave, which is similar to the behaviour exhibited by the wave systems in figures 9 and 5 ; a tail is also developing in the trailing portion of the wave system. At the last station of observation in figure 10, the leading soliton has separated entirely from the trailing wave structure as the water level on each side of the wave crest merges with the still-water level. (A theoretical soliton profile is shown superposed on the measured leading soliton at this station.) The second wave, denoted by 2 , is still progressing along the leading face of the negative wave preceding the oscillatory portion of the tail.

The results presented in figures 9 and 10 for two particular initial wave systems in which the net volume is zero indicate that solitons evolve from the large 
positive waves in the initial data, and progress to the front of the evolving wave train. The large negative waves in the initial data appear to coalesce into a tail (as the solitons emerge at the front of the wave system) which then evolves in the same manner as observed in figure 7.

\subsection{Comparison of leading-soliton amplitudes and $N$}

The experimental results presented in $\S \S 4.1,4.2$ and 4.3 demonstrate the evolution of solitons from various sets of initial data and the decay in amplitude of these solitons during propagation. This effect as well as the reduction in the centroid velocity of the evolving wave systems was mentioned briefly in $\S 4.1$ and was attributed to energy losses and boundary stresses resulting from the presence of viscosity in the experiments. Since the KdV equation is an inviscid model of wave propagation, the effects of viscosity on the experimental wave amplitudes must be estimated before quantitative comparisons with theory are meaningful. It is the objective of this sub-section to approximate the effects of viscosity on the amplitude of the leading soliton which evolves during an experiment and to compare the observed amplitude with the corrected theoretical amplitude found from (10) using the initial data as the potential.

Keulegan (1948) derived a theoretical model for the damping of a solitary wave due to viscous dissipation in the boundary layers adjacent to the solid boundaries of a wave tank. For a single solitary wave with an initial amplitude $\eta_{0 i}$ at $x=0$ in water of depth $h$ and a tank of width $W$, Keulegan found that the maximum amplitude $\eta_{0}$ of the solitary wave at any downstream position $x$ was approximately given by

$$
\begin{aligned}
& \frac{\eta_{0}}{h}=\left(\frac{\eta_{0 i}}{h}\right)\left[1+\theta\left(\frac{\eta_{0 i}}{h}\right)^{\frac{1}{4}}\right]^{-4} \\
& \theta=\frac{1}{12}\left(1+\frac{2 h}{W}\right)\left(\frac{\nu^{2}}{g h^{3}}\right)^{\frac{1}{5}}\left(\frac{x}{h}\right)
\end{aligned}
$$

where

and $\nu$ is the kinematic fluid viscosity. Laboratory experiments have been conducted by several authors to test the validity of (21). Some of these results are summarized by French (1969), who also includes data from his own experiments, which were conducted in the same wave tank as was used in the present study. The data of French agree well with (21), differing at most by $6 \%$; however, the range of $\theta$ investigated by French $(\theta \leqslant 0.021)$ was smaller than the range $(\theta \leqslant 0 \cdot 223)$ involved in the present study. The data of Ippen, Kulin \& Raza (1955) generally indicate that Keulegan's expression predicts more amplitude decay than is actually observed. Their results at a water depth of approximately $6 \mathrm{~cm}$ suggests that $(21)$ predicts the amplitude decay to within $12 \%$ for $\eta_{0 i} / h \leqslant 0 \cdot 5$. (The range of $\theta$ used by Ippen et al. cannot be determined from their presentation of their results; however, it does appear that their range of $\theta$ exceeded that of French.) The results of these authors indicate that (21) predicts the viscous decay of a solitary wave with acceptable accuracy. The waves considered in this study are much more complex than individual solitary waves; however, for the waves generated by monotonic uplifts of the piston such as those 
Korteweg-de Vries equation and water waves. Part 2

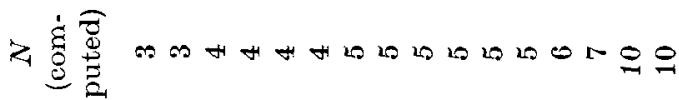

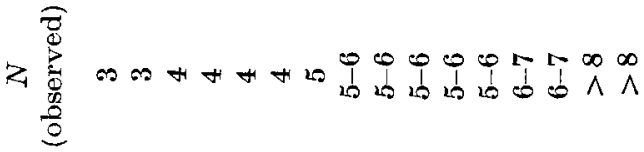

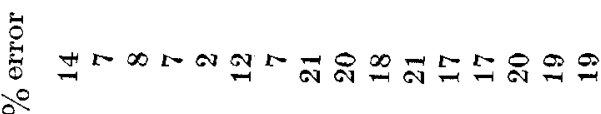

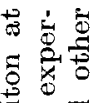

음

a. .5

$2^{\circ}$

हैं

똟

\& II

나의

$\mp \infty$

岁

눈

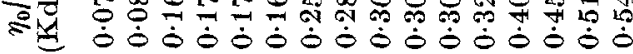

类 है है

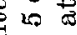

हा1

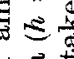

胥

要要

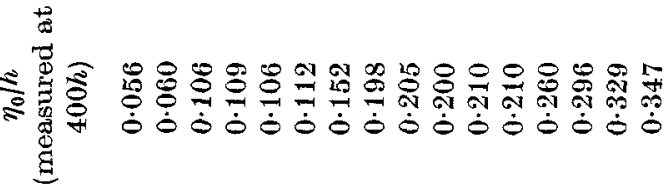

7.7

تृ

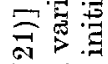

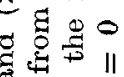

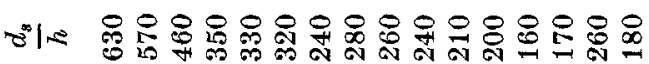

50.

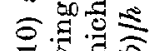

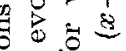

覀

然

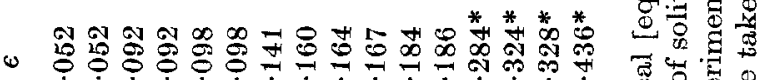


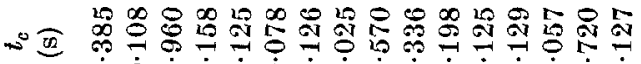

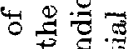
$\dot{0} \dot{0} \dot{0} \dot{0} \dot{0} \dot{0} \dot{0} \dot{0} \dot{0} \dot{0} \dot{0} \dot{0} \dot{0} \dot{0}$

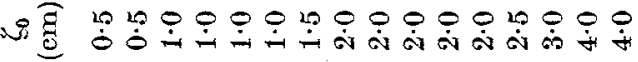

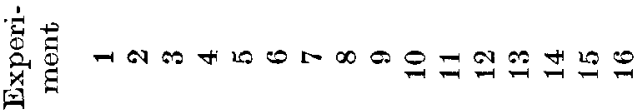




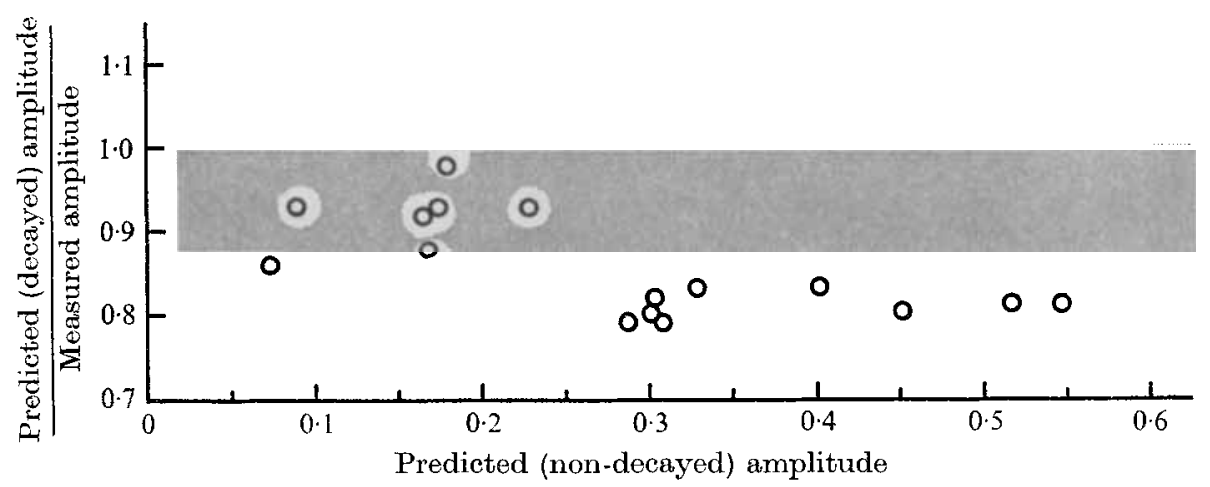

FigUre 11. Comparison of theoretical [equations (10) and (21)] and measured amplitudes of the leading soliton at $(x-b) / h=400$. Shaded area represents anticipated error range of equation (21).

shown in figure 3 and figure $6(a)$, the leading soliton rapidly develops at the front of the wave. This soliton is largely unaffected by the complexity of the rest of the wave system, and propagates into quiescent fluid as though it were an individual soliton. Hence, (21) will be used to estimate the viscous decay of the leading solitons (but no others) in the wave systems being considered.

Theoretical and experimental results are presented in table 1 and summarized in figure 11 for sixteen experiments with a uniform water depth of $5 \mathrm{~cm}$. The initial data in each experiment were generated by a monotonic uplift of the piston similar to the bed motion presented in figure $6(a)$. Hence, the initial wave systems for these experiments primarily consist of positive square waves similar to those presented in figures 3 and $6(a)$. The amplitude $\zeta_{0}$ of the bed motion and the total time $t_{c}$ of the bed displacement have been varied in order to vary the maximum amplitude and length of the initial data. The generation parameters $\zeta_{0}$ and $t_{c}$ for each experiment are shown in columns 2 and 3 , respectively, of table 1. The maximum amplitude $\epsilon=\left|\eta_{0}\right| / h$ of the initial data and the expected sorting distance $d_{s} / h$ are shown in columns 4 and 5 , respectively. (Note that the initial data in the last four experiments were chosen as the measured wave structure at $(x-b) / h=20$; this procedure was necessary in order to ensure that the initial data contained no water surface motions other than those caused by the passage of a free wave.) The measured amplitude of the leading soliton after 400 depths of propagation is shown in column 6 .

The theoretical amplitude of the leading soliton in the absence of viscous effects is computed from the initial data of each experiment using (10) and is listed in column 7 of table 1 . In order to correct the theoretical amplitudes in column 7 for viscous effects, these amplitudes are taken as the initial (nondecayed) amplitudes $\eta_{0 i} / h$ in Keulegan's equation (21), which is then solved for $\eta_{0} / h$ using $x / h=400(\theta=0 \cdot 223)$ in the first twelve experiments and $x / h=380$ $(\theta=0.212)$ in the last four. The results of these computations are listed in column 8 and represent the theoretical amplitude of the leading soliton corrected for viscosity. This procedure for predicting amplitudes of leading solitons necessarily assumes (i) that the dissipation of energy and the resulting decay in ampli- 
tude of the leading soliton are not significantly influenced by its nonlinear interaction with the rest of the wave; (ii) that the leading soliton has essentially completed its interaction with the rest of the wave after propagating 400 depths, so that the measured amplitude at this position truly represents the asymptotic wave structure; and (iii) that the error inherent in using temporal measurements to represent spatial data is negligible. The validity of the first assumption is unknown and this limitation must be remembered in interpreting the results. The validity of the second assumption is indicated by the computed sorting distance in column 5, which is less than 400 depths except for experiments 1,2 and 3 ; hence, this assumption does appear to be valid for most experiments. The validity of the third assumption is suggested by the magnitude of $\epsilon$ appearing in column 4 of table 1 since the error incurred is $O(\epsilon)$. For these experiments $\epsilon$ ranges from 0.052 in experiment 1 to 0.436 in experiment 16 ; hence, the error involved in the third assumption is expected to increase (and possibly become appreciable) in the last experiments of table 1.

The observed and the predicted amplitudes (columns 6 and 8, respectively) of the leading soliton for each experiment are compared in figure 11; the percentage error of the prediction for each experiment is shown in column 9 of table 1 . The predicted amplitudes in column 8 are less than the measured amplitudes in column 6 in all experiments, which is consistent with the previous results of Ippen et al. (1955). In the light of their work, errors of approximately $12 \%$ are anticipated as a result of the inaccuracy of (21). (An error band of this magnitude is shown in figure 11.) The observed errors in column 9 are within this range for most of the experiments in which the 'non-decayed' soliton amplitudes predicted by the $\mathrm{KdV}$ theory alone, i.e. column 7 or the abscissa in figure 11, do not exceed $0 \cdot 25$. (The non-decayed amplitude appears to be the best indicator in these experiments of the potential range of application of the $\mathrm{KdV}$ equation as a model of wave propagation in an inviscid fluid. A wave is not characterized by its measured amplitude at a particular location; this amplitude depends on the location, because of viscous effects. Nor is it characterized by the initial amplitude $\epsilon$, as can be seen from table 1 . Hence, the non-decayed amplitude is used in figure 11, even though it is not measured directly.) Even when these 'nondecayed' soliton amplitudes exceed half the still-water depth, discrepancies between theory and experiment never exceed about $20 \%$. It is not clear from this study where the increasing errors arise for experiments in which the soliton amplitudes predicted by the KdV theory exceed $0 \cdot 25$; possible sources are (i) a gradual breakdown of the KdV theory for large soliton amplitudes, (ii) the use of temporal wave records to represent spatial data, or (iii) a combination of these effects (assuming that the viscosity correction is in fact valid to within $12 \%$ as suggested by aforementioned publications). In any case, the suggested algorithm for predicting leading-soliton amplitudes in a viscous fluid is surprisingly accurate over the full range of initial data investigated.

The last two columns of table 1 indicate the number of observed solitons after 400 depths of propagation and the theoretical number computed from the initial data by numerically integrating (13). As the number of solitons in the initial data increases it becomes exceedingly difficult to count their exact number at the last 
station of observation owing to the small amplitudes of the solitons at the rear of the train. This difficulty is illustrated in column 10 of table 1 for the last nine experiments, where exact counts of $N$ were not possible. The theoretical results presented in column 11 agree exactly with the experimental counts in column 10 where exact counts were possible and fall within the range of the count in the last nine experiments.

It is worthwhile emphasizing in this discussion of soliton amplitudes that the experimental data indicate that the evolution of solitons from various sets of initial data does occur in a dissipative medium such as encountered in a laboratory wave tank. The amplitude of each soliton decays during propagation in the dissipative medium but the shape of the soliton remains self-similar. This behaviour is demonstrated by the leading soliton in figures 9 and 10, where theoretical profiles based on (11) are shown superposed on the measured waves at different downstream locations. Hence, the time scale over which significant viscous effects occur in the laboratory is such that the wave has ample time to adjust and retain the proper $\operatorname{sech}^{2}$ profile for the changing wave amplitude.

\section{Conclusions}

From the experimental results presented in this study, the following conclusions regarding the asymptotic behaviour of various sets of initial data propagating in a fluid of uniform depth can be made.

(i) When the net volume in the initial wave system is positive, waves of stable form (solitons) evolve, ordered by amplitude, whose shape is given by (11); these solitons are followed by a dispersive train of oscillatory waves. The number of solitons and their amplitudes depend on the initial data.

(ii) When the net volume in the initial wave system is negative, or zero, the evolution of solitons depends on the details of the initial data. If the initial data are non-positive everywhere, no solitons evolve.

(iii) The number of solitons that evolve from the initial data can be predicted by determining the number of discrete eigenvalues of the associated scattering problem using the initial wave system as the potential.

(iv) The dispersive train of oscillatory waves which evolves from most initial data can be described as slowly varying with the local wavenumber $k$, which increases towards the rear of the train from $k=0$ near the front of the train. The group velocity of this tail is negative relative to $(g h)^{\frac{1}{2}}$.

(v) After a sufficiently long time, the solitons are not affected by the tail, and vice versa.

(vi) High frequency content in the initial data has no permanent effect on the evolving solitons.

(vii) The amplitude of the leading soliton after it has propagated 400 depths is predicted to within about $20 \%$ by (10) and (21), over the entire range of initial data tested. If the ratio of the non-decayed amplitude predicted by (10) alone to the water depth does not exceed about $0 \cdot 25$, computations using (10) and (21) agree with the measured amplitudes to within the apparent accuracy $(12 \%)$ of $(21)$. 
(viii) The evolution of solitons is possible in a slightly dissipative fluid medium such as that encountered in a laboratory wave tank. The amplitude of each soliton slowly decreases during propagation; however, the shape of the soliton appears to have ample time to adjust to the changing amplitude so that the profile remains described by (11).

(ix) A sorting distance as defined by (19) appears to be adequate in approximating the distance of propagation required for the asymptotic development of initial data for which the number of evolving solitons is small.

In the light of the above conclusions, the Korteweg-de Vries equation appears to provide an accurate model for determining the evolution from various sets of initial data of gravity waves of moderate amplitude propagating in one direction in a non-dissipative or slightly dissipative fluid of uniform depth.

The authors gratefully acknowledge the kind assistance of the faculties and staffs of the Mathematics Department at Clarkson College of Technology and the W. M. Keck Laboratory of Hydraulics and Water Resources at the California Institute of Technology. This research was supported by the National Science Foundation under Grants GK-2370, GK-24716 and GA-27727A1 and the Office of Naval Research, U.S. Navy.

\section{REFERENCES}

ABLowitz, M. J. \& NewELL, A. C. 1973 The decay of the continuous spectrum for solutions of the Korteweg-de Vries equation. J. Math. Phys. 14, 1277-1284.

Benjamtn, T. B., Bona, J. L. \& Mahony, J. J. 1972 Model equations for long waves in nonlinear dispersive systems. Phil. Trans. 272, 47-78.

Benjamin, T. B. \& Mahony, J. J. 1971 On an invariant property of water waves. J. Fluid Mech. 49, 385-389.

BenNeY, D. J. \& Luke, J. C. 1964 On the interaction of permanent waves of finite amplitude. J. Math. \& Phys. 43, 309-313.

French, J. A. 1969 Wave uplift pressures on horizontal platforms. W. M. Keck Lab. Hydraul. \& Water Res., Calif. Inst. Tech. Rep. KH-R-19.

Gardner, C. S., Greene, J. M., Kruskal, M. D. \& Miura, R. M. 1967 Method for solving the Korteweg-de Vries equation. Phys, Rev. Lett. 19, 1095-1097.

Hammack, J. L. 1972 Tsunamis - a model of their generation and propagation. W. $M$. Keck Lab. Hydraul. \& Water Res., Calif. Inst. Tech. Rep. KH-R-28.

HАMмACK, J. L. 1973 A note on tsunamis: their generation and propagation in an ocean of uniform depth. J. Fluid Mech. 60, 769-799.

Hershrowitz, N., Romesser, T. \& Montgomery, D. 1972 Multiple soliton production and the Korteweg-de Vries equation. Phys. Rev. Lett. 29, 1586-1589.

IPPEN, A. T., KULIN, G. \& RAZA, M. A. 1955 Damping characteristics of the solitary wave. Hydrodyn. Lab. M.I.T. Tech. Rep. no. 16.

Keulegan, G. H. 1948 Gradual damping of solitary waves. J. Res. Nat. Bur. Stand.40, 487-498.

KORTEWEG, D. J. \& DE VRIEs, G. 1895 On the change of form of long waves advancing in a rectangular canal, and on a new type of long stationary waves. Phil. Mag. 39 (5), 422-443.

Miura, R. M., Gardner, C. S. \& Kruskal, M. D. 1968 Korteweg-de Vries equation and generalizations. II. Existence of conservation laws and constants of motion. $J$. Math. Phys. 9, 1204-1209. 
SEgUR, H. 1973 The Korteweg-de Vries equation and water waves. Part 1. Solutions of the equation. J. Fluid Mech. 59, 721-736.

Whitham, G. B. 1965 Nonlinear dispersive waves. Proc. Roy. Soc. A283, 238-261.

ZABUSkY, N.J. \& GALVIN, C.J. 1971 Shallow-water waves, the Korteweg-de Vries equation and solitons. J. Fluid Mech. 47, 811-824.

ZABUSKY, N. J. \& KRUSKaI, M. D. 1965 Interactions of 'solitons' in a collisionless plasma and the recurrence of initial states. Phys. Rev. Lett. 15, 240-243. 